\title{
Early History of the Determination of Atomic Charge
}

$\mathrm{A}^{\mathrm{T}}$

TOMICITY is a characteristic of twentieth century physies, so that modern physics may be said to begin with those early investigations by which the atomic nature of electricity was first established. The facts relating to early investigations of the atomic charge are scattered through the literature of the subject, and it will be of interest to bring them together in a brief account of the development of the methods which led to the accurate determination of this fundamental constant.

That electricity has an atomic structure appears first to have been suggested to explain Faraday's laws of electrolysis, on the supposition that the charge on a monovalent ion was the smallest separate quantity. This hypothesis was strongly advocated by Stoney and Helmholtz. It follows from Faraday's experiments that if $E$ be the charge on a monovalent ion in a liquid, and $n$ the number of molecules in a cubic centimetre of a gas at $15^{\circ} \mathrm{C}$. and $76 \mathrm{~cm}$. pressure, the product $n . E$ is $1.24 \times 10^{10}$, $E$ being expressed in electrostatic units. This $E$ is taken as the fundamental atomic charge.

The quantities $n$ and $E$ could not be deduced separately from experiments on electrolysis, and in the earliest attempts to determine $E$ the above value of $n . E$ was used with approximate estimates of $n$. All that was known about $n$ prior to a direct measurement of an atomic charge, was that "it lies between the limits $10^{18}$ and $10^{21}$ " as stated by Sir J. J. Thomson in his book "The Discharge of Electricity through Gases" published in 1898, where be also gives a description of the first experiments on the direct determination of the atomic charge made by Townsend, ${ }^{1}$ who was at that time demonstrator in the Cavendish Laboratory, Cambridge.

These experiments of Townsend are notable as being the prototype of the investigations which led finally to the accurate determination of the atomic charge made by Millikan.

It was known that in many cases electrically charged clouds were formed when newly prepared gases were bubbled through water. Townsend conceived the idea of measuring the charge on a drop in such a cloud, which he supposed to be an atomic charge, and for this purpose he used gases prepared by electrolysis, adopting the following procedure. The total weight $W$ of the drops in a cubic centimetre of the cloud, and the total charge $Q$ carried by them, were found by direct measurements, but in order to determine the average charge on a drop it was necessary to find the number of drops in a cubic centimetre of the cloud; to obtain this number the weight $w$ of a drop was required.

The weight of a drop and also its radius were obtained from the rate of fall of a cloud in a beaker, by an ingenious application of Stokes's formula for the velocity of a sphere moving slowly in a viscous medium under the action of a constant force. The first value found for an atomic charge was $3 \times 10^{-10}$ E.S.U., but after allowing for the fact that the cloud contained drops with charges of different sign, Townsend ${ }^{2}$ gave a corrected value $5 \times 10^{-10}$.

The importance of this investigation was immediately recognised, and in $1898 \mathrm{Sir}$ J. J. Thomson used the same method modified in details to investigate the charge carried by ions produced by $\mathrm{X}$-rays. In Thomson's experiments a cloud was formed in a closed vessel as in C. T. R. Wilson's experiments in which an ionised gas is cooled by a sudden expansion causing water vapour to condense on the ions. With this modification, it was necessary to determine the charge $Q$ per cubic centimetre and the weight $W$ indirectly, consequently $Q$ was obtained from the electrical conductivity of the gas and $W$ by estimating the amount of moisture condensed on ions by the expansion. ${ }^{3}$ The gases were ionised by X-rays and the values $6.5 \times 10^{-10}$ E.S.U. and $6.7 \times 10^{-10}$ E.S.U. found for the charge on a drop in air and hydrogen respectively. Later he obtained the value $6.8 \times 10^{-10}$ for negative ions generated by the action of ultra-violet light. ${ }^{4}$ In a subsequent determination $^{5}$ which he considered to be more accurate, he used a radioactive substance to ionise the gas, obtaining for the value of the atomic charge $3.4 \times 10^{-10}$.

The determinations of Townsend and Thomson involve various inaccuracies, and also the serious uncertainty in the assumption that each drop in the cloud carried a single atomic charge. Millikan states that, in the clouds produced in ionised gases by expansion, some of the drops may carry one, some two, some ten or almost any number of unit charges, though this does not imply that before condensation the ions in the gas have multiple charges.

There remained, in addition, the difficulty of establishing a relation between the charge $e$ on an ion in a gas and the charge $E$ on a monovalent ion in an electrolyte. A method of showing that these charges are identical was first given by Townsend ${ }^{6}$, who showed that if $e$ be the charge on an ion in a gas, $U$ its mean velocity in the direction of an electric force $Z$, and $K$ the coefficient of diffusion of the ions, then the value of $n . e$ is obtained from the equation $U / K=$ n.e. $Z / P$ where $P$ is the pressure of a gas containing $n$ molecules per cubic centimetre at the temperature at which $U$ and $K$ are deter. mined ( $P=10^{8}$ C.G.S. units)

In order to find the product n.e, Townsend devised a method by which he measured the coefficient $K$, and taking the mean values of $K$ for moist and dry gases with the corresponding values of $U$ obtained by Rutherford, he found the following values for $n . e \times 10^{-10}$ : air $1 \cdot 35$; oxygen $1 \cdot 25$; carbon dioxide $1 \cdot 30$; hydrogen $1 \cdot 00$.

These values of n.e were so near the value $1.24 \times 10^{10}$ of $n . E$ for monovalent ions in a liquid that Townsend was able definitely to state that 
the charge on ions produced by $\mathrm{X}$-rays and the charge $E$ on a monovalent ion in a liquid are the same. In later investigations in which the ions were produced in various ways, he found that the value of $n$.e for negative ions in gases was in close agreement with the value of $n . E$ for electro. lytic ions.

Further progress in the accurate determination of $e$ was made when $\mathrm{H}$. A. Wilson introduced an important improvement into the method of finding the charge on a drop, whereby it was possible to avoid the uncertainty of dealing with drops with an unknown number of atomic charges. The cloud was formed in the ionised gas by expansion, in an apparatus containing two horizontal parallel electrodes, so that it was possible to establish a vertical field $Z$ in the space where the cloud was formed. A retarding or an accelerating force, $Z$.e, was thus superimposed on the force of gravity $w$ acting on each drop. The weight $w$ of a drop was determined by measuring the velocity when $Z$ was zero and by applying Stokes's formula as in the original method. The charge $e$ was determined by finding the change in the velocity when the force $Z . e$ and $w$ acted together. In this method it was unnecessary to find the number of drops in the cloud. It was observed that the change in the velocity due to the electric force was not the same for each drop, since the cloud appeared to fall in groups of drops with charges in the proportion $1: 2: 3$.

This important observation showed that the charge on a drop, if not equal to, was a multiple of the smallest charge. H. A. Wilson gave $3 \cdot 1 \times 10^{-10}$ as the value of the atomic charge according to this method."

It is well known that the most accurate determinations of the atomic charge were made by Millikan in the period 1909-1916.8 As it was unnecessary to find the number of drops in the cloud in order to determine $e$, Millikan applied
H. A. Wilson's method to single drops. Inaccuracies due to evaporation were avoided by using drops of non-volatile oil, which were placed in electric fields of such strength that they could be made to move against gravity or held in suspension at will. A single drop could thus be kept under observation for long periods. $\mathrm{He}$ tested Stokes's formula, which had been assumed by his predecessors, obtaining an empirical correction to it in the case of small drops, and his very consistent results ${ }^{8}$ showed that the charges carried by drops were always exact multiples of the atomic charge $4 \cdot 77 \times 10^{-10}$ E.S.U.

The earliest investigations of the atomic charge had an important bearing on the interpretation of the experiments made to determine the ratio of the charge $e$ to the mass $m$ of an electron. The first trustworthy determinations of the ratio $\mathrm{e} / \mathrm{m}$ were made in 1897 by Wiechert, ${ }^{9}$ Kaufmann, ${ }^{10}$ and Thomson, ${ }^{11}$ who found that the ratio $e / m$ for cathode rays was about $1 / 2000$ of the ratio $E / M$ of the atomic charge $E$ to the mass $M$ of an atom of hydrogen. It was known from experiments on electrolysis that the ratio $E / M$ was $2.9 \times 10^{14}$ while the best experiments gave $5 \cdot 3 \times 10^{12}$ for the ratio $e / m$, and from the first it was suggested by Wiechert that the charges $E$ and $e$ were equal and consequently that the mass $m$ was about $M / 2000$. This suggestion, however, could only be regarded as a plauisible hypothesis until the identity of the charges $E$ and $e$ had been established in 1899, in relation to which Townsend's investigation of the product n.e ranks in importance with the accurate determination of the atomic charge $e$.

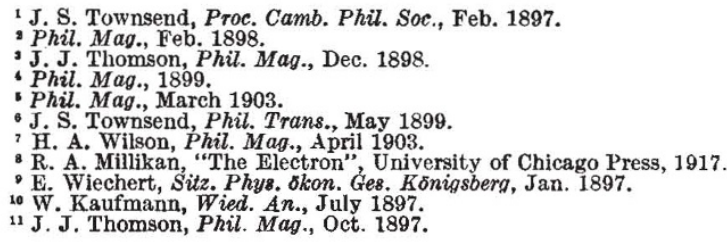

\section{Scientific and Industrial Research*}

$\mathrm{T}$ HE seventeenth annual report of the Department of Scientific and Industrial Research covering the period August 1, 1931-July 31, 1932, appears appropriately as if to punctuate the tributes to the importance of research in our national life which have recently been paid by high authorities in finance. A more impressive picture of the manifold ways in which scientific research is daily applied not merely to the solution of our industrial problems but also to the service of the innumerable needs of a civilised community than is contained in the annual reports of this Department it is difficult to imagine. The present report, including the brief report of the Privy Council, signed by the Right Hon. Stanley Baldwin, and the longer report of the Advisory Council,

* Department of Scientific and Industrial Research. Report for the Year 1931-32. (Cmd. 4254.) Pp. iv + 193, (London: H.M. Stationery Office, 1933.) 38. net. over Lord Rutherford's signature, is no exception to the rule. Lucid as are these reports and the summaries of the work carried out by the National Physical Laboratory, the Chemical Research Laboratory, the research associations, and under the direction of some forty-five research boards and committees, a popular exposition of the matter contained in them should be invaluable propaganda and assist the ordinary citizen to appreciate the magnitude of the contribution thus made to the necessities and luxuries of his daily. life-food and clothing, air and water supply, transport and housing, business and pleasure. Appendices to the report deal with finance, publications and the personnel of the various boards and committees.

There is no department of State in which the beneficial influence of the Department of Scientific and Industrial Research is not felt and no 